CRYSTALLOGRAPHIC COMMUNICATIONS

ISSN 2056-9890

Received 1 July 2020

Accepted 8 July 2020

Edited by H. Ishida, Okayama University, Japan

Keywords: crystal structure; 8-quinolinol ; bridging ligand; tridentate ligand; $\mathrm{C}-\mathrm{H} \cdots \pi$ interactions.

CCDC reference: 2014831

Supporting information: this article has supporting information at journals.iucr.org/e

\section{Crystal structure of 7,7'-[(pyridin-2-yl)methylene]- bis(5-chloroquinolin-8-ol)}

\author{
Yukiyasu Kashiwagi, ${ }^{a *}$ Koji Kubono ${ }^{b}$ and Toshiyuki Tamai ${ }^{a}$

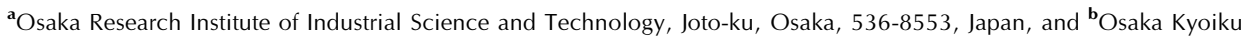 \\ University, Kashiwara, Osaka 582-8582, Japan. *Correspondence e-mail: kasiwagi@omtri.or.jp
}

In the title compound, $\mathrm{C}_{24} \mathrm{H}_{15} \mathrm{Cl}_{2} \mathrm{~N}_{3} \mathrm{O}_{2}$, one quinoline ring system is essentially planar and the other is slightly bent. An intramolecular $\mathrm{O}-\mathrm{H} \cdots \mathrm{N}$ hydrogen bond involving the hydroxy group and a pyridine $\mathrm{N}$ atom forms an $S(5)$ ring motif. In the crystal, two molecules are associated into an inversion dimer with two $R_{2}^{2}(7)$ ring motifs through intermolecular $\mathrm{O}-\mathrm{H} \cdots \mathrm{N}$ and $\mathrm{O}-\mathrm{H} \cdots \mathrm{O}$ hydrogen bonds. The dimers are further linked by an intermolecular $\mathrm{C}-\mathrm{H} \cdots \mathrm{O}$ hydrogen bond and four $\mathrm{C}-\mathrm{H} \cdots \pi$ interactions, forming a two-dimensional network parallel to (001).

\section{Chemical context}

8-Quinolinol and its derivatives are well-known chelating agents in analytical chemistry and bidentate ligands to metal ions in structural chemistry. Recently, multinuclear metal complexes based on the dimeric 8-quinolinol ligand, 1,1-bis(8hydroxyquinolin-7-yl)ethane, have been investigated (Zhu et al., 2012; Zhang et al., 2014; Wu et al., 2017; Gao et al., 2018). On the other hand, Yamato et al. $(1986,1987)$ reported the aromatic-group-substituted dimeric 8-quinolinol derivatives, 1,1-bis(8-hydroxyquinolin-7-yl)-1-(4-methoxyphenyl)methane, 1,1-bis(8-hydroxyquinolin-7-yl)-1-(furan-2-yl)methane and 1,1-bis(8-hydroxyquinolin-7-yl)-1-(thiophen-2-yl)methane, to be candidates for antitumor agents. We are attempting to develop a 2-pyridyl group-introduced dimeric 8-quinolinolbased ligand for mono- and multi-nuclear metal complexes, and report here the crystal structure of the title compound.<smiles>Oc1c(C(c2ccccn2)c2cc(Cl)c3cccnc3c2O)cc(Cl)c2cccnc12</smiles>

\section{Structural commentary}

The molecular structure of the title compound is shown in Fig. 1. One quinoline ring system is essentially planar, the dihedral angle between the mean planes through C22-C24/N6 and $\mathrm{C} 26 / \mathrm{C} 18-\mathrm{C} 20$ being $0.5(2)^{\circ}$. The other quinoline ring system is slightly bent, the dihedral angle between the mean planes through $\mathrm{N} 5 / \mathrm{C} 10-\mathrm{C} 12$ and $\mathrm{C} 14-\mathrm{C} 16 / \mathrm{C} 8$ being 5.77 (18) ${ }^{\circ}$. 
Table 1

Hydrogen-bond geometry $\left(\AA,^{\circ}\right)$.

$C g 1, C g 2$ and $C g 3$ are the centroids of the $\mathrm{C} 18-\mathrm{C} 21 / \mathrm{C} 25 / \mathrm{C} 26, \mathrm{C} 8 / \mathrm{C} 9 / \mathrm{C} 13-\mathrm{C} 16$ and $\mathrm{N} 7 / \mathrm{C} 27-\mathrm{C} 31$ rings, respectively.

\begin{tabular}{lllll}
\hline$D-\mathrm{H} \cdots A$ & $D-\mathrm{H}$ & $\mathrm{H} \cdots A$ & $D \cdots A$ & $D-\mathrm{H} \cdots A$ \\
\hline $\mathrm{O} 3-\mathrm{H} 3 \cdots \mathrm{N} 5$ & $0.82(2)$ & $2.23(3)$ & $2.727(2)$ & $119(2)$ \\
$\mathrm{O} 3-\mathrm{H} 3 \cdots \mathrm{O} 4^{\mathrm{i}}$ & $0.83(3)$ & $2.26(3)$ & $3.044(2)$ & $159(2)$ \\
$\mathrm{O} 4-\mathrm{H} 4 \cdots \mathrm{N} 5^{\mathrm{i}}$ & $0.84(4)$ & $2.16(3)$ & $2.878(3)$ & $144(2)$ \\
$\mathrm{O} 4-\mathrm{H} 4 \cdots \mathrm{N} 6$ & $0.84(4)$ & $2.20(4)$ & $2.674(3)$ & $115(3)$ \\
$\mathrm{C} 29-\mathrm{H} 29 \cdots \mathrm{O} 3^{\text {ii }}$ & 0.95 & 2.53 & $3.377(3)$ & 148 \\
$\mathrm{C} 10-\mathrm{H} 10 \cdots C g 1^{\text {iii }}$ & 0.95 & 2.75 & $3.659(3)$ & 161 \\
$\mathrm{C} 22-\mathrm{H} 22 \cdots C g 2^{\text {iv }}$ & 0.95 & 2.92 & $3.667(3)$ & 136 \\
$\mathrm{C} 24-\mathrm{H} 24 \cdots C g 3^{\text {v }}$ & 0.95 & 2.65 & $3.528(3)$ & 153 \\
$\mathrm{C} 30-\mathrm{H} 30 \cdots C g 1^{\text {ii }}$ & 0.95 & 2.73 & $3.562(3)$ & 147
\end{tabular}

Symmetry codes: (i) $-x+1,-y+2,-z+2$; (ii) $x-1, y, z$; (iii) $x+1, y+1, z$; (iv) $x, y-1, z ;(\mathrm{v})-x,-y+1,-z+2$.

There are two intramolecular $\mathrm{O}-\mathrm{H} \cdots \mathrm{N}$ hydrogen bonds involving the hydroxy groups and quinoline $\mathrm{N}$ atoms $(\mathrm{O} 3-$ $\mathrm{H} 3 \cdots \mathrm{N} 5$ and $\mathrm{O} 4-\mathrm{H} 4 \cdots \mathrm{N} 6$; Table 1) generating $S(5)$ ring motifs (Fig. 1). The arrangement of the 2-pyridyl and two quinoline rings is propeller-wise, which is a common arrangement for $\mathrm{Ar}_{3} \mathrm{C}-\mathrm{H}$ fragments. The bond angles $\mathrm{C} 16-$ $\mathrm{C} 17-\mathrm{C} 18, \mathrm{C} 16-\mathrm{C} 17-\mathrm{C} 27$, and $\mathrm{C} 18-\mathrm{C} 17-\mathrm{C} 27$ are $112.21(16), 112.64(16)$ and $112.94(16)^{\circ}$, respectively. The torsion angles $\mathrm{C} 8-\mathrm{C} 16-\mathrm{C} 17-\mathrm{C} 18, \mathrm{C} 26-\mathrm{C} 18-\mathrm{C} 17-\mathrm{C} 27$, and $\mathrm{C} 28-\mathrm{C} 27-\mathrm{C} 17-\mathrm{C} 16$ are $-88.6(2),-101.2(2)$ and $-87.8(2)^{\circ}$, respectively.

\section{Supramolecular features}

In the crystal, molecules are linked by intermolecular $\mathrm{O}-$ $\mathrm{H} \cdots \mathrm{O}$ and $\mathrm{O}-\mathrm{H} \cdots \mathrm{N}$ hydrogen bonds $\left[\mathrm{O} 3-\mathrm{H} 3 \cdots \mathrm{O} 4^{\mathrm{i}}\right.$ and O4-H4 $\cdots \mathrm{N} 5$; symmetry code: (i) $-x+1,-y+2,-z+2]$, forming an inversion dimer with two $R_{2}^{2}(7)$ ring motifs (Fig. 2 and Table 1). In contrast, the crystal structure of 1,1-bis(8hydroxyquinolin-7-yl)methane, an analogue of the title

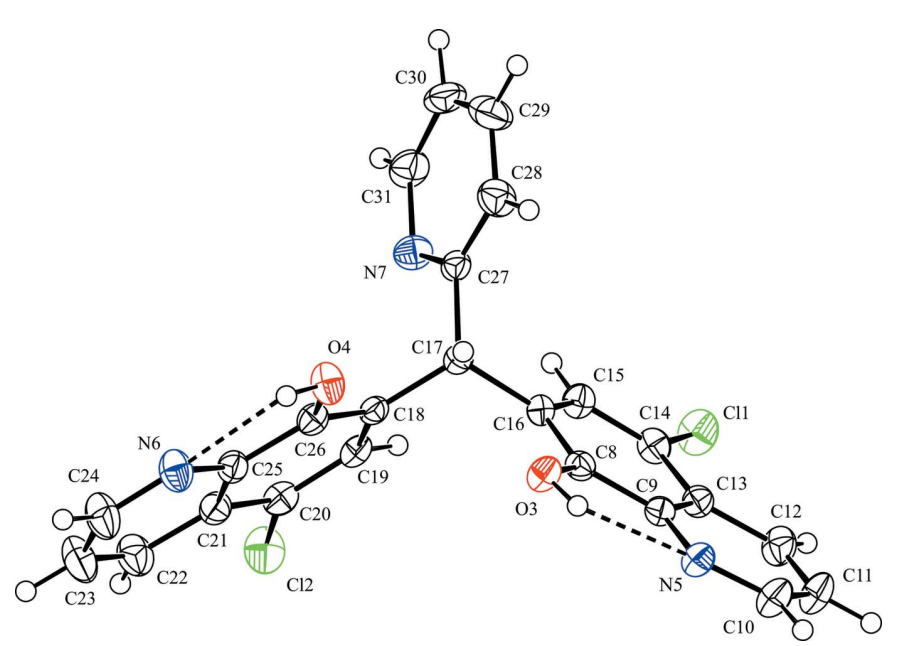

Figure 1

The molecular structure of the title compound, with the atom labelling. Displacement ellipsoids are drawn at the $50 \%$ probability level. $\mathrm{H}$ atoms are represented by spheres of arbitrary radius. The intramolecular $\mathrm{O}-$ $\mathrm{H} \cdots \mathrm{N}$ hydrogen bonds are shown as dashed lines.

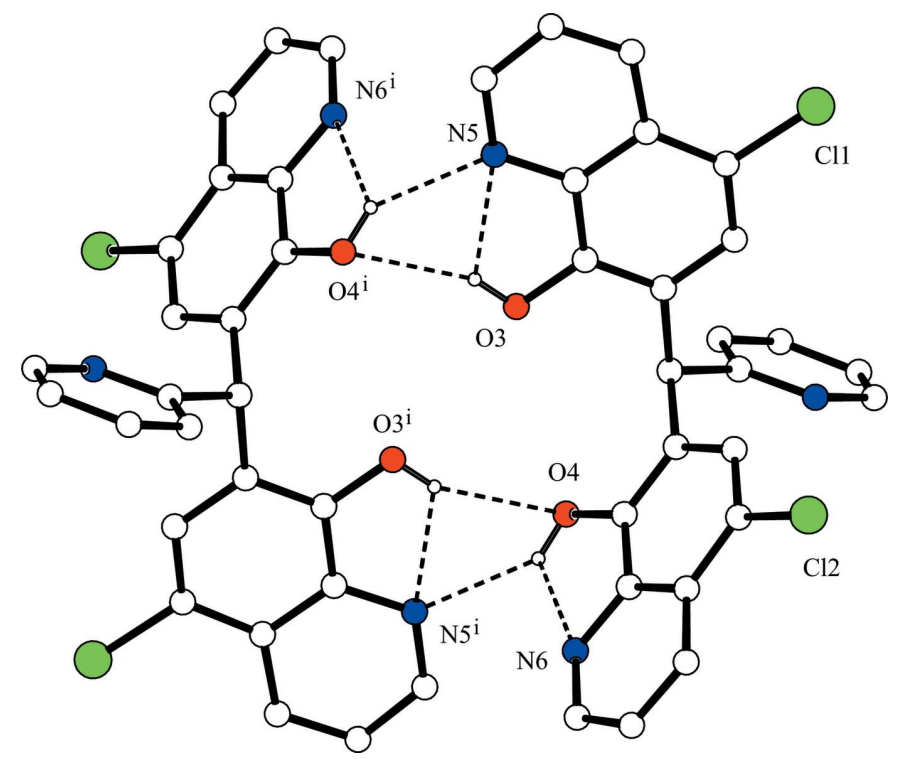

Figure 2

A centrosymmetric dimeric structure of the title compound. The intraand intermolecular hydrogen bonds are shown as dashed lines. $\mathrm{H}$ atoms not involved in these interactions have been omitted for clarity. [Symmetry code: (i) $-x+1,-y+2,-z+2$.]

compound with the 2-pyridyl group omitted, exhibits a supramolecular 1D-polymeric structure with intermolecular hydrogen bonding between each 8-quinolinol unit and two other molecules (CSD refcode CIBCEV; Albrecht et al., 1999). The dimers of the title compound are linked by complementary $\mathrm{C}-\mathrm{H} \cdots \pi$ interactions $\left[\mathrm{C} 10-\mathrm{H} 10 \cdots C g 1^{\mathrm{iii}}\right.$ and $\mathrm{C} 24-$ $\mathrm{H} 24 \cdot \cdots C g 3^{\mathrm{v}} ; C g 1$ is the centroid of the $\mathrm{C} 18-\mathrm{C} 21 / \mathrm{C} 25 / \mathrm{C} 26$ ring and $C g 3$ is the centroid of the N7/C27-C31 ring; symmetry codes: (iii) $x+1, y+1, z$; (v) $-x,-y+1,-z+2$ ], forming a ribbon structure along [110] (Fig. 3). Considered separately, the 1D-chain structure propagates along the $a$-axis direction through a $\mathrm{C}-\mathrm{H} \cdots \mathrm{O}$ hydrogen bond $\left[\mathrm{C} 29-\mathrm{H} 29 \cdots \mathrm{O} 3^{\mathrm{ii}}\right.$;

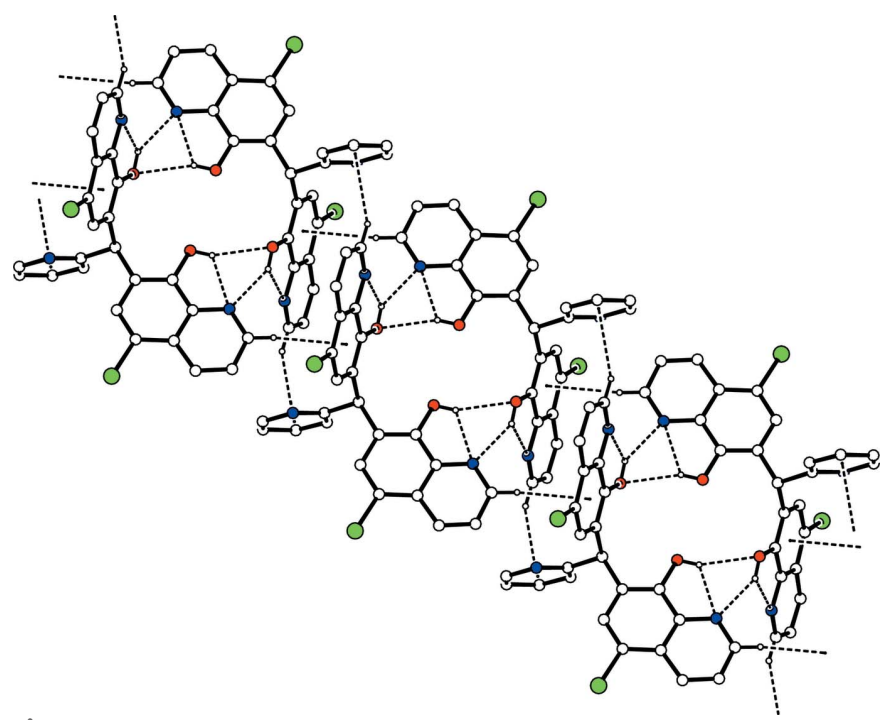

Figure 3

A packing diagram of the title compound, showing the ribbon structure. The $\mathrm{C}-\mathrm{H} \cdots \pi$ interactions between the dimers are shown as dashed lines. $\mathrm{H}$ atoms not involved in the interactions have been omitted for clarity. 


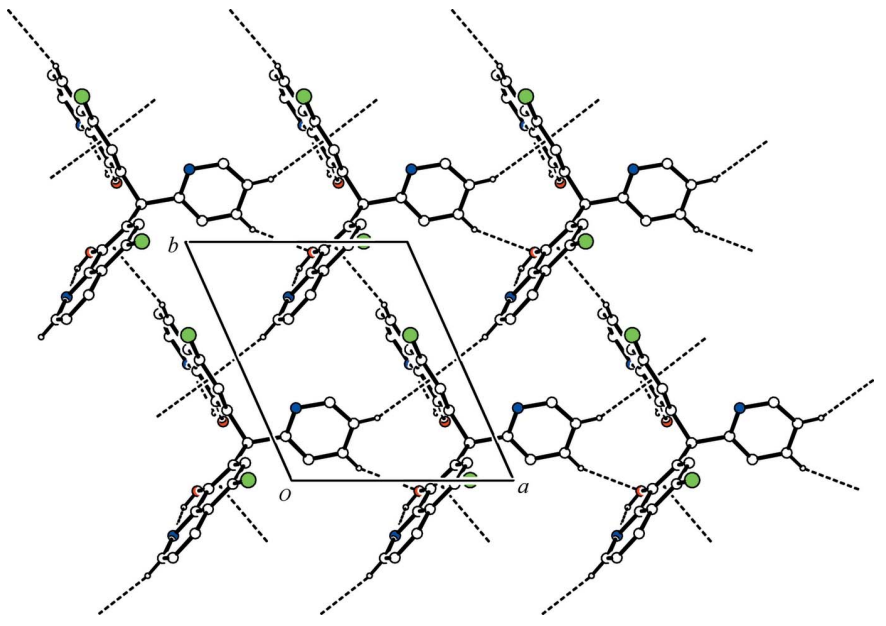

Figure 4

A packing diagram of the title compound viewed along the $c$ axis, showing the two-dimensional network sheet structure. The $\mathrm{C}-\mathrm{H} \cdots \pi$ interactions and $\mathrm{C}-\mathrm{H} \cdots \mathrm{O}$ hydrogen bonds are shown as dashed lines. $\mathrm{H}$ atoms not involved in the interactions have been omitted for clarity.

symmetry code: (ii) $x-1, y, z]$ and a $\mathrm{C}-\mathrm{H} \cdots \pi$ interaction [C30-H30 $\cdots C g 1^{\text {ii }} ; C g 1$ is the centroid of the C18-C21/C25/ $\mathrm{C} 26$ ring]. The chains are linked by two $\mathrm{C}-\mathrm{H} \cdots \pi$ interactions $\left[\mathrm{C} 10-\mathrm{H} 10 \cdots C g 1^{\mathrm{iii}}\right.$ and $\mathrm{C} 22-\mathrm{H} 22 \cdots C g 2^{\mathrm{iv}} ; \quad C g 2$ is the centroid of the $\mathrm{C} 8 / \mathrm{C} 9 / \mathrm{C} 13-\mathrm{C} 16$ ring; symmetry code: (iv) $x, y-$ $1, z]$, generating a two-dimensional network parallel to (001) (Fig. 4).

\section{Database survey}

A search of the Cambridge Structural Database (CSD, Version 5.41, update of March 2020; Groom et al., 2016) for compounds containing the bis(phenol-2-yl)methane skeleton gave 9360 hits, and for those containing the 8-quinolinol skeleton gave 3200 hits. A search for the fragment of 1,1-bis(8hydroxyquinolin-7-yl)methane gave 23 hits (21 compounds), which included only one organic compound, 1,1-bis(8-hydroxyquinolin-7-yl)methane (CIBCEV; Albrecht et al., 1999), and 20 metal complexes with 1,1-bis(8-hydroxyquinolin-7yl)ethane as bridging ligands. The 20 metal complexes include two dinuclear complexes, $\mathrm{Zn}_{2}$ (Wu et al., 2017; Gao et al., 2018) and $\mathrm{Cd}_{2}$ (Gao et al., 2018), one homo-trinuclear $\mathrm{La}_{3}$ complex (Wu et al., 2017), 16 hetero-trinuclear complexes, $\mathrm{Co}_{2} \mathrm{Sm}$, $\mathrm{Ni}_{2} \mathrm{Sm}, \mathrm{Zn}_{2} \mathrm{Sm}, \mathrm{Co}_{2} \mathrm{Eu}, \mathrm{Ni}_{2} \mathrm{Eu}, \mathrm{Zn}_{2} \mathrm{Eu}, \mathrm{Cd}_{2} \mathrm{Eu}, \mathrm{Co}_{2} \mathrm{Gd}, \mathrm{Ni}_{2} \mathrm{Gd}$, $\mathrm{Cd}_{2} \mathrm{Gd}, \mathrm{Co}_{2} \mathrm{~Tb}, \mathrm{Ni}_{2} \mathrm{~Tb}, \mathrm{Zn}_{2} \mathrm{~Tb}, \mathrm{Fe}_{2} \mathrm{Dy}, \mathrm{Co}_{2} \mathrm{Dy}, \mathrm{Cd}_{2} \mathrm{Dy}$ (Zhu et al., 2012) and one hexanuclear $\mathrm{Na}_{2} \mathrm{Co}_{4}$ complex (Zhang et al., 2014). The crystal structure of 1,1-bis(8-hydroxyquinolin-7yl)ethane itself has not been reported.

\section{Synthesis and crystallization}

The title compound was prepared by a modification of the reported $\mathrm{K}_{2} \mathrm{CO}_{3}$-catalysed synthetic method for 1,1-bis(5chloro-8-hydroxyquinolin-7-yl)methane (Ozawa \& Shibuya, $1963 a, b)$. 5-Chloro-8-hydroxyquinoline (898 mg, $5.0 \mathrm{mmol}$ ), 2pyridinecarboxaldehyde (321 mg, $3.0 \mathrm{mmol}), \mathrm{K}_{2} \mathrm{CO}_{3}(100 \mathrm{mg}$,
Table 2

Experimental details.

\begin{tabular}{|c|c|}
\hline \multicolumn{2}{|l|}{ Crystal data } \\
\hline Chemical formula & $\mathrm{C}_{24} \mathrm{H}_{15} \mathrm{Cl}_{2} \mathrm{~N}_{3} \mathrm{O}_{2}$ \\
\hline$M_{\mathrm{r}}$ & 448.31 \\
\hline Crystal system, space group & Triclinic, $P \overline{1}$ \\
\hline Temperature $(\mathrm{K})$ & 173 \\
\hline$a, b, c(\AA)$ & $\begin{array}{l}8.7077(8), 10.4281(10) \\
\quad 12.1329(11)\end{array}$ \\
\hline$\alpha, \beta, \gamma\left({ }^{\circ}\right)$ & $101.111(7), 92.087(7), 113.161(8)$ \\
\hline$V\left(\AA^{3}\right)$ & $986.23(17)$ \\
\hline$Z$ & 2 \\
\hline Radiation type & Мо $K \alpha$ \\
\hline$\mu\left(\mathrm{mm}^{-1}\right)$ & 0.36 \\
\hline Crystal size $(\mathrm{mm})$ & $0.40 \times 0.15 \times 0.10$ \\
\hline \multicolumn{2}{|l|}{ Data collection } \\
\hline Diffractometer & Rigaku R-AXIS RAPID \\
\hline Absorption correction & $\begin{array}{l}\text { Multi-scan (ABSCOR; Higashi, } \\
\text { 1995) }\end{array}$ \\
\hline$T_{\min }, T_{\max }$ & $0.710,0.965$ \\
\hline $\begin{array}{l}\text { No. of measured, independent and } \\
\text { observed }\left[F^{2}>2.0 \sigma\left(F^{2}\right)\right] \text { reflec- } \\
\text { tions }\end{array}$ & $9484,4475,3536$ \\
\hline$R_{\mathrm{int}}$ & 0.027 \\
\hline$(\sin \theta / \lambda)_{\max }\left(\AA^{-1}\right)$ & 0.649 \\
\hline \multicolumn{2}{|l|}{ Refinement } \\
\hline$R\left[F^{2}>2 \sigma\left(F^{2}\right)\right], w R\left(F^{2}\right), S$ & $0.050,0.119,1.07$ \\
\hline No. of reflections & 4475 \\
\hline No. of parameters & 288 \\
\hline H-atom treatment & $\begin{array}{l}\mathrm{H} \text { atoms treated by a mixture of } \\
\text { independent and constrained } \\
\text { refinement }\end{array}$ \\
\hline$\Delta \rho_{\max }, \Delta \rho_{\min }\left(\mathrm{e} \AA^{-3}\right)$ & $0.43,-0.22$ \\
\hline
\end{tabular}

Computer programs: RAPID-AUTO (Rigaku, 2006), SIR92 (Altomare et al., 1993), SHELXL2014/7 (Sheldrick, 2015), PLATON (Spek, 2020) and CrystalStructure (Rigaku, 2016).

$0.72 \mathrm{mmol})$ and ethanol $(6 \mathrm{~mL})$ were placed in a $15 \mathrm{~mL}$ capped pressure tube. It was heated at $353 \mathrm{~K}$ for $96 \mathrm{~h}$. The generated pale-white precipitate was filtered to give a palewhite solid ( $806 \mathrm{mg}, 1.80 \mathrm{mmol}$; yield $72 \%$ ). Single crystals of title compound suitable for X-ray diffraction were grown by slow evaporation of a solution in $\mathrm{CHCl}_{3} / n$-hexane $(2: 1, v / v)$ at ambient temperature. ${ }^{1} \mathrm{H}$ NMR $\left(\mathrm{CDCl}_{3}, 600 \mathrm{MHz}\right) \delta=6.63(s$, $1 \mathrm{H}), 7.21(d d d, 1 \mathrm{H}, J=7.8,4.8,1.8 \mathrm{~Hz}), 7.33(d, 1 \mathrm{H}, J=7.8 \mathrm{~Hz})$, $7.52(s, 2 \mathrm{H}), 7.52(d d, 2 \mathrm{H}, J=8.4,4.2 \mathrm{~Hz}), 7.67(t d, 1 \mathrm{H}, J=7.8$, $1.8 \mathrm{~Hz}), 8.48(d d, 2 \mathrm{H}, J=8.4,1.2 \mathrm{~Hz}), 8.64(d, 1 \mathrm{H}, J=4.8 \mathrm{~Hz})$, $8.81(d d, 2 \mathrm{H}, J=4.2,1.2 \mathrm{~Hz}), 8.84(b r, 2 \mathrm{H})$.

\section{Refinement}

Crystal data, data collection and structure refinement details are summarized in Table 2. Hydroxy $\mathrm{H}$ atoms were located in a difference-Fourier map and freely refined. C-bound $\mathrm{H}$ atoms were placed in geometrically calculated positions $(\mathrm{C}-\mathrm{H}=$ 0.95-1.00 ̊) and refined as part of a riding model with $U_{\text {iso }}(\mathrm{H})$ $=1.2 U_{\mathrm{eq}}(\mathrm{C})$. One outlier (111) was omitted from the refinement.

\section{Funding information}

Funding for this research was provided by: JSPS KAKENHI (grant No. JP20K05565). 


\section{References}

Albrecht, M., Blau, O., Witt, K., Wegelius, E., Nissinen, M., Risssanen, K. \& Fröhlich, R. (1999). Synthesis, pp. 1819-1829.

Altomare, A., Cascarano, G., Giacovazzo, C. \& Guagliardi, A. (1993). J. Appl. Cryst. 26, 343-350.

Gao, X., Xu, W., Wu, C.-L., Zhu, X.-M., Ou, Y.-C. \& Wu, J.-Z. (2018). Chin. J. Inorg. Chem. 34, 1768-1774.

Groom, C. R., Bruno, I. J., Lightfoot, M. P. \& Ward, S. C. (2016). Acta Cryst. B72, 171-179.

Higashi, T. (1995). ABSCOR. Rigaku Corporation, Tokyo, Japan.

Ozawa, T. \& Shibuya, K. (1963a). Yakugaku Zasshi, 83, 498-502.

Ozawa, T. \& Shibuya, K. (1963b). Chem. Abstr. 59, 7481.

Rigaku (2006). RAPID-AUTO. Rigaku Corporation, Tokyo, Japan.

Rigaku (2016). CrystalStructure. Rigaku Corporation, Tokyo, Japan.
Sheldrick, G. M. (2015). Acta Cryst. C71, 3-8.

Spek, A. L. (2020). Acta Cryst. E76, 1-11.

Wu, M.-M., Wang, J.-Y., Sun, R., Zhao, C., Zhao, J.-P., Che, G.-B. \& Liu, F.-C. (2017). Inorg. Chem. 56, 9555-9562.

Yamato, M., Hashigaki, K., Yasumoto, Y., Sakai, J., Luduena, R. F., Banerjee, A., Tsukagoshi, S., Tashiro, T. \& Tsuruo, T. (1987). J. Med. Chem. 30, 1897-1900.

Yamato, M., Hashigaki, K., Yasumoto, Y., Sakai, J., Tsukagoshi, S., Tashiro, T. \& Tsuruo, T. (1986). Chem. Pharm. Bull. 34, 34963498.

Zhang, X.-M., Li, J.-Q., Liu, S.-J., Luo, M.-B., Xu, W.-Y. \& Luo, F. (2014). CrystEngComm, 16, 2570-2573.

Zhu, Y., Luo, F., Song, Y.-M., Feng, X.-F., Luo, M.-B., Liao, Z.-W., Sun, G.-M., Tian, X.-Z. \& Yuan, Z.-J. (2012). Cryst. Growth Des. 12, 2158-2161. 


\section{supporting information}

Acta Cryst. (2020). E76, 1271-1274 [https://doi.org/10.1107/S2056989020009317]

\section{Crystal structure of 7,7'-[(pyridin-2-yl)methylene]bis(5-chloroquinolin-8-ol)}

\section{Yukiyasu Kashiwagi, Koji Kubono and Toshiyuki Tamai}

Computing details

Data collection: RAPID-AUTO (Rigaku, 2006); cell refinement: RAPID-AUTO (Rigaku, 2006); data reduction: RAPIDAUTO (Rigaku, 2006); program(s) used to solve structure: SIR92 (Altomare et al., 1993); program(s) used to refine structure: SHELXL2014/7 (Sheldrick, 2015); molecular graphics: PLATON (Spek, 2020); software used to prepare material for publication: CrystalStructure (Rigaku, 2016).

7,7'-[(pyridin-2-yl)methylene]bis(5-chloroquinolin-8-ol)

Crystal data

$\mathrm{C}_{24} \mathrm{H}_{15} \mathrm{Cl}_{2} \mathrm{~N}_{3} \mathrm{O}_{2}$

$M_{r}=448.31$

Triclinic, $P \overline{1}$

$a=8.7077(8) \AA$

$b=10.4281(10) \AA$

$c=12.1329(11) \AA$

$\alpha=101.111(7)^{\circ}$

$\beta=92.087(7)^{\circ}$

$\gamma=113.161(8)^{\circ}$

$V=986.23(17) \AA^{3}$

\section{Data collection}

Rigaku R-AXIS RAPID diffractometer

Detector resolution: 10.000 pixels $\mathrm{mm}^{-1}$

$\omega$ scans

Absorption correction: multi-scan

(ABSCOR; Higashi, 1995)

$T_{\min }=0.710, T_{\max }=0.965$

9484 measured reflections

\section{Refinement}

Refinement on $F^{2}$

$R\left[F^{2}>2 \sigma\left(F^{2}\right)\right]=0.050$

$w R\left(F^{2}\right)=0.119$

$S=1.07$

4475 reflections

288 parameters

0 restraints

Primary atom site location: structure-invariant direct methods
$Z=2$

$F(000)=460.00$

$D_{\mathrm{x}}=1.510 \mathrm{Mg} \mathrm{m}^{-3}$

Mo $K \alpha$ radiation, $\lambda=0.71075 \AA$

Cell parameters from 7587 reflections

$\theta=3.0-27.5^{\circ}$

$\mu=0.36 \mathrm{~mm}^{-1}$

$T=173 \mathrm{~K}$

Chunk, colorless

$0.40 \times 0.15 \times 0.10 \mathrm{~mm}$

4475 independent reflections

3536 reflections with $F^{2}>2.0 \sigma\left(F^{2}\right)$

$R_{\text {int }}=0.027$

$\theta_{\max }=27.5^{\circ}, \theta_{\min }=3.1^{\circ}$

$h=-11 \rightarrow 11$

$k=-13 \rightarrow 13$

$l=-15 \rightarrow 15$

Secondary atom site location: difference Fourier map

Hydrogen site location: inferred from neighbouring sites

$\mathrm{H}$ atoms treated by a mixture of independent and constrained refinement

$w=1 /\left[\sigma^{2}\left(F_{0}^{2}\right)+(0.0532 P)^{2}+0.572 P\right]$ where $P=\left(F_{\mathrm{o}}^{2}+2 F_{\mathrm{c}}^{2}\right) / 3$

$(\Delta / \sigma)_{\max }<0.001$

$\Delta \rho_{\max }=0.43 \mathrm{e}^{-3}$

$\Delta \rho_{\min }=-0.22$ e $\AA^{-3}$ 


\section{Special details}

Geometry. All esds (except the esd in the dihedral angle between two 1.s. planes) are estimated using the full covariance matrix. The cell esds are taken into account individually in the estimation of esds in distances, angles and torsion angles; correlations between esds in cell parameters are only used when they are defined by crystal symmetry. An approximate (isotropic) treatment of cell esds is used for estimating esds involving l.s. planes.

Refinement. Refinement was performed using all reflections. The weighted R-factor (wR) and goodness of fit (S) are based on $\mathrm{F}^{2}$. R-factor (gt) are based on $\mathrm{F}$. The threshold expression of $\mathrm{F}^{2}>2.0 \operatorname{sigma}\left(\mathrm{F}^{2}\right)$ is used only for calculating Rfactor (gt).

Fractional atomic coordinates and isotropic or equivalent isotropic displacement parameters $\left(\AA^{2}\right)$

\begin{tabular}{|c|c|c|c|c|}
\hline & $x$ & $y$ & $z$ & $U_{\text {iso }} * / U_{\text {eq }}$ \\
\hline $\mathrm{Cl1}$ & $0.19486(7)$ & $0.99797(7)$ & $0.42458(5)$ & $0.03826(17)$ \\
\hline $\mathrm{Cl} 2$ & $0.17365(8)$ & $0.39091(6)$ & $0.56639(5)$ & $0.03966(17)$ \\
\hline $\mathrm{O} 3$ & 0.44767 (19) & $1.04439(17)$ & $0.89332(12)$ & $0.0268(3)$ \\
\hline $\mathrm{O} 4$ & $0.1923(2)$ & $0.75295(16)$ & $1.00827(13)$ & $0.0305(3)$ \\
\hline N5 & $0.6459(2)$ & $1.23152(19)$ & $0.77548(15)$ & 0.0269 (4) \\
\hline N6 & $0.2352(3)$ & $0.5120(2)$ & $1.00448(15)$ & $0.0328(4)$ \\
\hline N7 & $-0.1639(2)$ & $0.6963(2)$ & $0.71721(16)$ & $0.0306(4)$ \\
\hline $\mathrm{C} 8$ & $0.3918(2)$ & $1.0343(2)$ & $0.78420(16)$ & $0.0216(4)$ \\
\hline $\mathrm{C} 9$ & $0.4946(2)$ & $1.1297(2)$ & $0.72083(16)$ & $0.0220(4)$ \\
\hline $\mathrm{C} 10$ & $0.7377(3)$ & $1.3256(2)$ & $0.7197(2)$ & $0.0340(5)$ \\
\hline $\mathrm{H} 10$ & 0.8414 & 1.3988 & 0.7577 & $0.041^{*}$ \\
\hline C11 & 0.6909 (3) & $1.3234(3)$ & $0.6073(2)$ & $0.0367(5)$ \\
\hline H11 & 0.7630 & 1.3927 & 0.5706 & $0.044^{*}$ \\
\hline $\mathrm{C} 12$ & $0.5418(3)$ & $1.2218(2)$ & $0.55110(19)$ & $0.0322(5)$ \\
\hline $\mathrm{H} 12$ & 0.5093 & 1.2188 & 0.4747 & $0.039 *$ \\
\hline $\mathrm{C} 13$ & $0.4351(3)$ & $1.1202(2)$ & $0.60802(17)$ & $0.0246(4)$ \\
\hline $\mathrm{C} 14$ & $0.2728(3)$ & $1.0141(2)$ & $0.56284(17)$ & $0.0260(4)$ \\
\hline C15 & 0.1743 (3) & 0.9261 (2) & $0.62662(17)$ & $0.0257(4)$ \\
\hline H15 & 0.0646 & 0.8576 & 0.5945 & $0.031^{*}$ \\
\hline $\mathrm{C} 16$ & $0.2326(2)$ & $0.9353(2)$ & $0.73891(16)$ & 0.0217 (4) \\
\hline $\mathrm{C} 17$ & $0.1239(2)$ & $0.8422(2)$ & 0.81349 (17) & $0.0218(4)$ \\
\hline H17 & 0.1639 & 0.8989 & 0.8932 & $0.026^{*}$ \\
\hline $\mathrm{C} 18$ & $0.1510(2)$ & $0.7059(2)$ & $0.80759(16)$ & 0.0207 (4) \\
\hline C19 & $0.1464(2)$ & $0.6152(2)$ & $0.70280(17)$ & 0.0235 (4) \\
\hline H19 & 0.1239 & 0.6396 & 0.6343 & $0.028^{*}$ \\
\hline $\mathrm{C} 20$ & $0.1734(3)$ & $0.4941(2)$ & $0.69780(17)$ & $0.0261(4)$ \\
\hline $\mathrm{C} 21$ & $0.2042(3)$ & $0.4507(2)$ & $0.79740(18)$ & $0.0263(4)$ \\
\hline $\mathrm{C} 22$ & $0.2328(3)$ & $0.3278(3)$ & $0.8022(2)$ & $0.0369(5)$ \\
\hline $\mathrm{H} 22$ & 0.2312 & 0.2638 & 0.7344 & $0.044^{*}$ \\
\hline $\mathrm{C} 23$ & $0.2627(4)$ & $0.3015(3)$ & $0.9047(2)$ & $0.0436(6)$ \\
\hline $\mathrm{H} 23$ & 0.2833 & 0.2196 & 0.9091 & $0.052 *$ \\
\hline $\mathrm{C} 24$ & $0.2627(3)$ & $0.3965(3)$ & 1.0039 (2) & $0.0401(6)$ \\
\hline $\mathrm{H} 24$ & 0.2839 & 0.3763 & 1.0746 & $0.048^{*}$ \\
\hline $\mathrm{C} 25$ & $0.2070(2)$ & $0.5402(2)$ & $0.90232(17)$ & $0.0243(4)$ \\
\hline $\mathrm{C} 26$ & $0.1822(2)$ & $0.6680(2)$ & $0.90558(17)$ & $0.0231(4)$ \\
\hline $\mathrm{C} 27$ & $-0.0611(2)$ & $0.8146(2)$ & $0.79228(16)$ & $0.0227(4)$ \\
\hline
\end{tabular}




\begin{tabular}{lllll}
$\mathrm{C} 28$ & $-0.1171(3)$ & $0.9116(2)$ & $0.8521(2)$ & $0.0342(5)$ \\
$\mathrm{H} 28$ & -0.0398 & 0.9966 & 0.9023 & $0.041^{*}$ \\
$\mathrm{C} 29$ & $-0.2853(3)$ & $0.8838(3)$ & $0.8384(2)$ & $0.0414(6)$ \\
$\mathrm{H} 29$ & -0.3263 & 0.9482 & 0.8799 & $0.050^{*}$ \\
$\mathrm{C} 30$ & $-0.3934(3)$ & $0.7608(3)$ & $0.7633(2)$ & $0.0390(6)$ \\
$\mathrm{H} 30$ & -0.5106 & 0.7378 & 0.7530 & $0.047^{*}$ \\
$\mathrm{C} 31$ & $-0.3284(3)$ & $0.6725(3)$ & $0.7037(2)$ & $0.0356(5)$ \\
$\mathrm{H} 31$ & -0.4031 & 0.5899 & 0.6498 & $0.043^{*}$ \\
$\mathrm{H} 3$ & $0.541(3)$ & $1.112(3)$ & $0.909(2)$ & $0.030(7)^{*}$ \\
$\mathrm{H} 4$ & $0.214(4)$ & $0.718(3)$ & $1.060(3)$ & $0.052(9)^{*}$ \\
\hline
\end{tabular}

Atomic displacement parameters $\left(\AA^{2}\right)$

\begin{tabular}{|c|c|c|c|c|c|c|}
\hline & $U^{11}$ & $U^{22}$ & $U^{33}$ & $U^{12}$ & $U^{13}$ & $U^{23}$ \\
\hline $\mathrm{Cl1}$ & $0.0417(3)$ & $0.0466(3)$ & $0.0227(3)$ & $0.0125(3)$ & $-0.0028(2)$ & $0.0125(2)$ \\
\hline $\mathrm{Cl} 2$ & 0.0575 (4) & 0.0339 (3) & $0.0246(3)$ & $0.0183(3)$ & $0.0078(2)$ & $0.0000(2)$ \\
\hline $\mathrm{O} 3$ & $0.0222(8)$ & $0.0304(8)$ & $0.0230(7)$ & $0.0050(7)$ & $-0.0010(6)$ & $0.0086(6)$ \\
\hline $\mathrm{O} 4$ & $0.0446(9)$ & $0.0283(8)$ & $0.0195(7)$ & $0.0161(7)$ & $0.0020(6)$ & $0.0051(6)$ \\
\hline N5 & $0.0237(9)$ & $0.0269(9)$ & $0.0270(9)$ & $0.0066(7)$ & $0.0032(7)$ & $0.0071(7)$ \\
\hline N6 & $0.0452(11)$ & $0.0309(10)$ & $0.0254(9)$ & $0.0176(9)$ & $0.0036(8)$ & $0.0088(8)$ \\
\hline N7 & $0.0249(9)$ & $0.0330(10)$ & $0.0300(10)$ & $0.0097(8)$ & 0.0007 (7) & $0.0033(8)$ \\
\hline $\mathrm{C} 8$ & $0.0229(10)$ & $0.0233(9)$ & $0.0208(9)$ & $0.0112(8)$ & $0.0030(7)$ & $0.0058(8)$ \\
\hline C9 & $0.0225(9)$ & $0.0219(9)$ & $0.0226(10)$ & $0.0102(8)$ & $0.0043(7)$ & $0.0049(8)$ \\
\hline $\mathrm{C} 10$ & $0.0273(11)$ & $0.0328(12)$ & $0.0346(12)$ & $0.0032(10)$ & $0.0044(9)$ & $0.0107(10)$ \\
\hline C11 & $0.0352(12)$ & $0.0351(12)$ & $0.0362(13)$ & $0.0058(10)$ & $0.0110(10)$ & $0.0176(10)$ \\
\hline $\mathrm{C} 12$ & 0.0395 (13) & $0.0355(12)$ & $0.0244(11)$ & $0.0150(10)$ & $0.0086(9)$ & $0.0130(9)$ \\
\hline $\mathrm{C} 13$ & $0.0269(10)$ & $0.0250(10)$ & $0.0238(10)$ & $0.0117(9)$ & $0.0059(8)$ & $0.0070(8)$ \\
\hline C14 & $0.0312(11)$ & $0.0284(10)$ & $0.0196(10)$ & $0.0136(9)$ & $0.0007(8)$ & $0.0054(8)$ \\
\hline $\mathrm{C} 15$ & $0.0247(10)$ & $0.0246(10)$ & $0.0245(10)$ & $0.0072(8)$ & $-0.0002(8)$ & $0.0046(8)$ \\
\hline $\mathrm{C} 16$ & $0.0229(10)$ & $0.0206(9)$ & $0.0232(10)$ & $0.0091(8)$ & $0.0036(7)$ & $0.0075(8)$ \\
\hline $\mathrm{C} 17$ & $0.0198(9)$ & $0.0225(9)$ & $0.0215(9)$ & $0.0063(8)$ & $0.0020(7)$ & $0.0063(7)$ \\
\hline $\mathrm{C} 18$ & $0.0164(9)$ & $0.0209(9)$ & $0.0231(10)$ & $0.0051(8)$ & $0.0028(7)$ & $0.0064(8)$ \\
\hline C19 & $0.0203(9)$ & $0.0259(10)$ & $0.0208(9)$ & $0.0045(8)$ & $0.0034(7)$ & $0.0077(8)$ \\
\hline $\mathrm{C} 20$ & $0.0256(10)$ & $0.0268(10)$ & $0.0208(10)$ & $0.0071(9)$ & $0.0040(8)$ & $0.0018(8)$ \\
\hline $\mathrm{C} 21$ & $0.0254(10)$ & $0.0245(10)$ & $0.0280(11)$ & $0.0084(8)$ & $0.0053(8)$ & $0.0067(8)$ \\
\hline $\mathrm{C} 22$ & $0.0492(14)$ & $0.0313(11)$ & $0.0344(12)$ & $0.0212(11)$ & $0.0090(10)$ & $0.0058(10)$ \\
\hline $\mathrm{C} 23$ & $0.0650(17)$ & $0.0361(13)$ & $0.0433(14)$ & $0.0318(13)$ & $0.0115(12)$ & 0.0145 (11) \\
\hline $\mathrm{C} 24$ & $0.0578(16)$ & $0.0375(13)$ & $0.0341(13)$ & $0.0254(12)$ & $0.0057(11)$ & $0.0154(11)$ \\
\hline $\mathrm{C} 25$ & $0.0235(10)$ & $0.0244(10)$ & $0.0249(10)$ & $0.0084(8)$ & $0.0030(8)$ & $0.0083(8)$ \\
\hline $\mathrm{C} 26$ & $0.0222(10)$ & $0.0223(9)$ & $0.0214(9)$ & $0.0061(8)$ & $0.0027(7)$ & $0.0036(8)$ \\
\hline $\mathrm{C} 27$ & $0.0210(9)$ & $0.0251(10)$ & $0.0229(10)$ & $0.0086(8)$ & $0.0035(7)$ & $0.0095(8)$ \\
\hline $\mathrm{C} 28$ & $0.0341(12)$ & $0.0300(11)$ & $0.0366(13)$ & $0.0137(10)$ & $0.0016(9)$ & $0.0025(9)$ \\
\hline C29 & $0.0428(14)$ & $0.0507(15)$ & $0.0446(14)$ & $0.0324(12)$ & $0.0123(11)$ & $0.0122(12)$ \\
\hline $\mathrm{C} 30$ & $0.0205(11)$ & $0.0566(15)$ & $0.0473(14)$ & 0.0167 (11) & $0.0079(9)$ & $0.0257(12)$ \\
\hline C31 & $0.0244(11)$ & $0.0373(12)$ & $0.0388(13)$ & $0.0067(10)$ & $-0.0035(9)$ & $0.0086(10)$ \\
\hline
\end{tabular}


Geometric parameters $\left(\AA,{ }^{\circ}\right)$

\begin{tabular}{|c|c|c|c|}
\hline $\mathrm{Cl1}-\mathrm{C} 14$ & $1.738(2)$ & $\mathrm{C} 16-\mathrm{C} 17$ & $1.528(3)$ \\
\hline $\mathrm{C} 12-\mathrm{C} 20$ & $1.744(2)$ & $\mathrm{C} 17-\mathrm{C} 18$ & $1.519(3)$ \\
\hline $\mathrm{O} 3-\mathrm{C} 8$ & $1.364(2)$ & $\mathrm{C} 17-\mathrm{C} 27$ & $1.524(3)$ \\
\hline $\mathrm{O} 3-\mathrm{H} 3$ & $0.82(3)$ & $\mathrm{C} 17-\mathrm{H} 17$ & 1.0000 \\
\hline $\mathrm{O} 4-\mathrm{C} 26$ & $1.360(2)$ & $\mathrm{C} 18-\mathrm{C} 26$ & $1.373(3)$ \\
\hline $\mathrm{O} 4-\mathrm{H} 4$ & $0.84(3)$ & $\mathrm{C} 18-\mathrm{C} 19$ & $1.420(3)$ \\
\hline $\mathrm{N} 5-\mathrm{C} 10$ & $1.319(3)$ & $\mathrm{C} 19-\mathrm{C} 20$ & $1.364(3)$ \\
\hline $\mathrm{N} 5-\mathrm{C} 9$ & $1.366(3)$ & C19-H19 & 0.9500 \\
\hline N6-C24 & $1.316(3)$ & $\mathrm{C} 20-\mathrm{C} 21$ & $1.421(3)$ \\
\hline $\mathrm{N} 6-\mathrm{C} 25$ & $1.363(3)$ & $\mathrm{C} 21-\mathrm{C} 22$ & $1.410(3)$ \\
\hline $\mathrm{N} 7-\mathrm{C} 27$ & $1.338(3)$ & $\mathrm{C} 21-\mathrm{C} 25$ & $1.419(3)$ \\
\hline N7-C31 & $1.352(3)$ & $\mathrm{C} 22-\mathrm{C} 23$ & $1.362(3)$ \\
\hline $\mathrm{C} 8-\mathrm{C} 16$ & $1.374(3)$ & $\mathrm{C} 22-\mathrm{H} 22$ & 0.9500 \\
\hline $\mathrm{C} 8-\mathrm{C} 9$ & $1.423(3)$ & $\mathrm{C} 23-\mathrm{C} 24$ & $1.403(4)$ \\
\hline $\mathrm{C} 9-\mathrm{C} 13$ & $1.417(3)$ & $\mathrm{C} 23-\mathrm{H} 23$ & 0.9500 \\
\hline $\mathrm{C} 10-\mathrm{C} 11$ & $1.402(3)$ & $\mathrm{C} 24-\mathrm{H} 24$ & 0.9500 \\
\hline $\mathrm{C} 10-\mathrm{H} 10$ & 0.9500 & $\mathrm{C} 25-\mathrm{C} 26$ & $1.425(3)$ \\
\hline $\mathrm{C} 11-\mathrm{C} 12$ & $1.360(3)$ & $\mathrm{C} 27-\mathrm{C} 28$ & $1.385(3)$ \\
\hline C11-H11 & 0.9500 & $\mathrm{C} 28-\mathrm{C} 29$ & $1.373(3)$ \\
\hline $\mathrm{C} 12-\mathrm{C} 13$ & $1.420(3)$ & $\mathrm{C} 28-\mathrm{H} 28$ & 0.9500 \\
\hline $\mathrm{C} 12-\mathrm{H} 12$ & 0.9500 & $\mathrm{C} 29-\mathrm{C} 30$ & $1.378(4)$ \\
\hline $\mathrm{C} 13-\mathrm{C} 14$ & $1.415(3)$ & $\mathrm{C} 29-\mathrm{H} 29$ & 0.9500 \\
\hline $\mathrm{C} 14-\mathrm{C} 15$ & $1.371(3)$ & $\mathrm{C} 30-\mathrm{C} 31$ & $1.369(4)$ \\
\hline $\mathrm{C} 15-\mathrm{C} 16$ & 1.409 (3) & $\mathrm{C} 30-\mathrm{H} 30$ & 0.9500 \\
\hline $\mathrm{C} 15-\mathrm{H} 15$ & 0.9500 & $\mathrm{C} 31-\mathrm{H} 31$ & 0.9500 \\
\hline $\mathrm{C} 8-\mathrm{O} 3-\mathrm{H} 3$ & $106.4(17)$ & $\mathrm{C} 19-\mathrm{C} 18-\mathrm{C} 17$ & $121.94(17)$ \\
\hline $\mathrm{C} 26-\mathrm{O} 4-\mathrm{H} 4$ & $110(2)$ & $\mathrm{C} 20-\mathrm{C} 19-\mathrm{C} 18$ & $121.80(18)$ \\
\hline $\mathrm{C} 10-\mathrm{N} 5-\mathrm{C} 9$ & $117.43(18)$ & $\mathrm{C} 20-\mathrm{C} 19-\mathrm{H} 19$ & 119.1 \\
\hline $\mathrm{C} 24-\mathrm{N} 6-\mathrm{C} 25$ & $117.4(2)$ & $\mathrm{C} 18-\mathrm{C} 19-\mathrm{H} 19$ & 119.1 \\
\hline $\mathrm{C} 27-\mathrm{N} 7-\mathrm{C} 31$ & $117.0(2)$ & $\mathrm{C} 19-\mathrm{C} 20-\mathrm{C} 21$ & $121.58(19)$ \\
\hline $\mathrm{O} 3-\mathrm{C} 8-\mathrm{C} 16$ & $118.84(17)$ & $\mathrm{C} 19-\mathrm{C} 20-\mathrm{Cl} 2$ & $119.47(16)$ \\
\hline $\mathrm{O} 3-\mathrm{C} 8-\mathrm{C} 9$ & $120.08(17)$ & $\mathrm{C} 21-\mathrm{C} 20-\mathrm{Cl} 2$ & $118.94(16)$ \\
\hline $\mathrm{C} 16-\mathrm{C} 8-\mathrm{C} 9$ & $121.02(18)$ & $\mathrm{C} 22-\mathrm{C} 21-\mathrm{C} 25$ & $116.8(2)$ \\
\hline $\mathrm{N} 5-\mathrm{C} 9-\mathrm{C} 13$ & $123.10(17)$ & $\mathrm{C} 22-\mathrm{C} 21-\mathrm{C} 20$ & $126.4(2)$ \\
\hline $\mathrm{N} 5-\mathrm{C} 9-\mathrm{C} 8$ & $116.96(17)$ & $\mathrm{C} 25-\mathrm{C} 21-\mathrm{C} 20$ & $116.81(19)$ \\
\hline $\mathrm{C} 13-\mathrm{C} 9-\mathrm{C} 8$ & $119.88(17)$ & $\mathrm{C} 23-\mathrm{C} 22-\mathrm{C} 21$ & 119.5 \\
\hline $\mathrm{N} 5-\mathrm{C} 10-\mathrm{C} 11$ & $123.6(2)$ & $\mathrm{C} 23-\mathrm{C} 22-\mathrm{H} 22$ & 120.3 \\
\hline $\mathrm{N} 5-\mathrm{C} 10-\mathrm{H} 10$ & 118.2 & $\mathrm{C} 21-\mathrm{C} 22-\mathrm{H} 22$ & 120.3 \\
\hline $\mathrm{C} 11-\mathrm{C} 10-\mathrm{H} 10$ & 118.2 & $\mathrm{C} 22-\mathrm{C} 23-\mathrm{C} 24$ & $119.4(2)$ \\
\hline $\mathrm{C} 12-\mathrm{C} 11-\mathrm{C} 10$ & $119.7(2)$ & $\mathrm{C} 22-\mathrm{C} 23-\mathrm{H} 23$ & 120.3 \\
\hline $\mathrm{C} 12-\mathrm{C} 11-\mathrm{H} 11$ & 120.1 & $\mathrm{C} 24-\mathrm{C} 23-\mathrm{H} 23$ & 120.3 \\
\hline $\mathrm{C} 10-\mathrm{C} 11-\mathrm{H} 11$ & 120.1 & $\mathrm{~N} 6-\mathrm{C} 24-\mathrm{C} 23$ & 123.7 (2) \\
\hline $\mathrm{C} 11-\mathrm{C} 12-\mathrm{C} 13$ & $119.2(2)$ & $\mathrm{N} 6-\mathrm{C} 24-\mathrm{H} 24$ & 118.1 \\
\hline $\mathrm{C} 11-\mathrm{C} 12-\mathrm{H} 12$ & 120.4 & $\mathrm{C} 23-\mathrm{C} 24-\mathrm{H} 24$ & 118.1 \\
\hline $\mathrm{C} 13-\mathrm{C} 12-\mathrm{H} 12$ & 120.4 & $\mathrm{~N} 6-\mathrm{C} 25-\mathrm{C} 21$ & $123.20(19)$ \\
\hline
\end{tabular}




\begin{tabular}{|c|c|c|c|}
\hline $\mathrm{C} 14-\mathrm{C} 13-\mathrm{C} 9$ & $117.62(17)$ & $\mathrm{N} 6-\mathrm{C} 25-\mathrm{C} 26$ & $116.15(19)$ \\
\hline $\mathrm{C} 14-\mathrm{C} 13-\mathrm{C} 12$ & $125.45(19)$ & $\mathrm{C} 21-\mathrm{C} 25-\mathrm{C} 26$ & $120.64(18)$ \\
\hline $\mathrm{C} 9-\mathrm{C} 13-\mathrm{C} 12$ & $116.90(19)$ & $\mathrm{O} 4-\mathrm{C} 26-\mathrm{C} 18$ & $120.59(18)$ \\
\hline $\mathrm{C} 15-\mathrm{C} 14-\mathrm{C} 13$ & $121.40(19)$ & $\mathrm{O} 4-\mathrm{C} 26-\mathrm{C} 25$ & $118.42(18)$ \\
\hline $\mathrm{C} 15-\mathrm{C} 14-\mathrm{Cl1}$ & $119.35(16)$ & $\mathrm{C} 18-\mathrm{C} 26-\mathrm{C} 25$ & $120.99(18)$ \\
\hline $\mathrm{C} 13-\mathrm{C} 14-\mathrm{Cl1}$ & $119.23(15)$ & $\mathrm{N} 7-\mathrm{C} 27-\mathrm{C} 28$ & $122.48(19)$ \\
\hline $\mathrm{C} 14-\mathrm{C} 15-\mathrm{C} 16$ & $121.20(18)$ & $\mathrm{N} 7-\mathrm{C} 27-\mathrm{C} 17$ & $118.58(18)$ \\
\hline $\mathrm{C} 14-\mathrm{C} 15-\mathrm{H} 15$ & 119.4 & $\mathrm{C} 28-\mathrm{C} 27-\mathrm{C} 17$ & $118.94(18)$ \\
\hline $\mathrm{C} 16-\mathrm{C} 15-\mathrm{H} 15$ & 119.4 & $\mathrm{C} 29-\mathrm{C} 28-\mathrm{C} 27$ & $119.5(2)$ \\
\hline $\mathrm{C} 8-\mathrm{C} 16-\mathrm{C} 15$ & $118.83(17)$ & $\mathrm{C} 29-\mathrm{C} 28-\mathrm{H} 28$ & 120.3 \\
\hline $\mathrm{C} 8-\mathrm{C} 16-\mathrm{C} 17$ & $118.67(17)$ & $\mathrm{C} 27-\mathrm{C} 28-\mathrm{H} 28$ & 120.3 \\
\hline $\mathrm{C} 15-\mathrm{C} 16-\mathrm{C} 17$ & $122.48(17)$ & $\mathrm{C} 28-\mathrm{C} 29-\mathrm{C} 30$ & $118.7(2)$ \\
\hline $\mathrm{C} 18-\mathrm{C} 17-\mathrm{C} 27$ & $112.94(16)$ & $\mathrm{C} 28-\mathrm{C} 29-\mathrm{H} 29$ & 120.7 \\
\hline $\mathrm{C} 18-\mathrm{C} 17-\mathrm{C} 16$ & $112.21(16)$ & $\mathrm{C} 30-\mathrm{C} 29-\mathrm{H} 29$ & 120.7 \\
\hline $\mathrm{C} 27-\mathrm{C} 17-\mathrm{C} 16$ & $112.64(16)$ & $\mathrm{C} 31-\mathrm{C} 30-\mathrm{C} 29$ & $118.7(2)$ \\
\hline $\mathrm{C} 18-\mathrm{C} 17-\mathrm{H} 17$ & 106.1 & $\mathrm{C} 31-\mathrm{C} 30-\mathrm{H} 30$ & 120.6 \\
\hline $\mathrm{C} 27-\mathrm{C} 17-\mathrm{H} 17$ & 106.1 & $\mathrm{C} 29-\mathrm{C} 30-\mathrm{H} 30$ & 120.6 \\
\hline $\mathrm{C} 16-\mathrm{C} 17-\mathrm{H} 17$ & 106.1 & $\mathrm{~N} 7-\mathrm{C} 31-\mathrm{C} 30$ & $123.6(2)$ \\
\hline $\mathrm{C} 26-\mathrm{C} 18-\mathrm{C} 19$ & $118.16(18)$ & $\mathrm{N} 7-\mathrm{C} 31-\mathrm{H} 31$ & 118.2 \\
\hline $\mathrm{C} 26-\mathrm{C} 18-\mathrm{C} 17$ & $119.90(17)$ & $\mathrm{C} 30-\mathrm{C} 31-\mathrm{H} 31$ & 118.2 \\
\hline $\mathrm{C} 10-\mathrm{N} 5-\mathrm{C} 9-\mathrm{C} 13$ & $1.1(3)$ & $\mathrm{C} 18-\mathrm{C} 19-\mathrm{C} 20-\mathrm{C} 21$ & $1.3(3)$ \\
\hline $\mathrm{C} 10-\mathrm{N} 5-\mathrm{C} 9-\mathrm{C} 8$ & $-176.2(2)$ & $\mathrm{C} 18-\mathrm{C} 19-\mathrm{C} 20-\mathrm{Cl} 2$ & $-177.68(15)$ \\
\hline $\mathrm{O} 3-\mathrm{C} 8-\mathrm{C} 9-\mathrm{N} 5$ & $-1.8(3)$ & $\mathrm{C} 19-\mathrm{C} 20-\mathrm{C} 21-\mathrm{C} 22$ & $179.7(2)$ \\
\hline $\mathrm{C} 16-\mathrm{C} 8-\mathrm{C} 9-\mathrm{N} 5$ & $175.27(19)$ & $\mathrm{C} 12-\mathrm{C} 20-\mathrm{C} 21-\mathrm{C} 22$ & $-1.3(3)$ \\
\hline $\mathrm{O} 3-\mathrm{C} 8-\mathrm{C} 9-\mathrm{C} 13$ & $-179.15(18)$ & $\mathrm{C} 19-\mathrm{C} 20-\mathrm{C} 21-\mathrm{C} 25$ & $-0.6(3)$ \\
\hline $\mathrm{C} 16-\mathrm{C} 8-\mathrm{C} 9-\mathrm{C} 13$ & $-2.0(3)$ & $\mathrm{C} 12-\mathrm{C} 20-\mathrm{C} 21-\mathrm{C} 25$ & $178.43(15)$ \\
\hline $\mathrm{C} 9-\mathrm{N} 5-\mathrm{C} 10-\mathrm{C} 11$ & $-2.1(3)$ & $\mathrm{C} 25-\mathrm{C} 21-\mathrm{C} 22-\mathrm{C} 23$ & $-0.6(3)$ \\
\hline N5- $110-\mathrm{C} 11-\mathrm{C} 12$ & $1.3(4)$ & $\mathrm{C} 20-\mathrm{C} 21-\mathrm{C} 22-\mathrm{C} 23$ & $179.2(2)$ \\
\hline $\mathrm{C} 10-\mathrm{C} 11-\mathrm{C} 12-\mathrm{C} 13$ & $0.6(4)$ & $\mathrm{C} 21-\mathrm{C} 22-\mathrm{C} 23-\mathrm{C} 24$ & $0.7(4)$ \\
\hline $\mathrm{N} 5-\mathrm{C} 9-\mathrm{C} 13-\mathrm{C} 14$ & $-177.04(19)$ & $\mathrm{C} 25-\mathrm{N} 6-\mathrm{C} 24-\mathrm{C} 23$ & $-0.7(4)$ \\
\hline $\mathrm{C} 8-\mathrm{C} 9-\mathrm{C} 13-\mathrm{C} 14$ & $0.1(3)$ & $\mathrm{C} 22-\mathrm{C} 23-\mathrm{C} 24-\mathrm{N} 6$ & $0.0(4)$ \\
\hline $\mathrm{N} 5-\mathrm{C} 9-\mathrm{C} 13-\mathrm{C} 12$ & $0.7(3)$ & $\mathrm{C} 24-\mathrm{N} 6-\mathrm{C} 25-\mathrm{C} 21$ & $0.7(3)$ \\
\hline $\mathrm{C} 8-\mathrm{C} 9-\mathrm{C} 13-\mathrm{C} 12$ & $177.9(2)$ & $\mathrm{C} 24-\mathrm{N} 6-\mathrm{C} 25-\mathrm{C} 26$ & $-178.3(2)$ \\
\hline $\mathrm{C} 11-\mathrm{C} 12-\mathrm{C} 13-\mathrm{C} 14$ & $176.0(2)$ & $\mathrm{C} 22-\mathrm{C} 21-\mathrm{C} 25-\mathrm{N} 6$ & $-0.1(3)$ \\
\hline $\mathrm{C} 11-\mathrm{C} 12-\mathrm{C} 13-\mathrm{C} 9$ & $-1.5(3)$ & $\mathrm{C} 20-\mathrm{C} 21-\mathrm{C} 25-\mathrm{N} 6$ & $-179.89(19)$ \\
\hline $\mathrm{C} 9-\mathrm{C} 13-\mathrm{C} 14-\mathrm{C} 15$ & $1.7(3)$ & $\mathrm{C} 22-\mathrm{C} 21-\mathrm{C} 25-\mathrm{C} 26$ & $178.89(19)$ \\
\hline $\mathrm{C} 12-\mathrm{C} 13-\mathrm{C} 14-\mathrm{C} 15$ & $-175.8(2)$ & $\mathrm{C} 20-\mathrm{C} 21-\mathrm{C} 25-\mathrm{C} 26$ & $-0.9(3)$ \\
\hline $\mathrm{C} 9-\mathrm{C} 13-\mathrm{C} 14-\mathrm{C} 11$ & $-179.77(16)$ & $\mathrm{C} 19-\mathrm{C} 18-\mathrm{C} 26-\mathrm{O} 4$ & $178.27(17)$ \\
\hline $\mathrm{C} 12-\mathrm{C} 13-\mathrm{C} 14-\mathrm{Cl1}$ & $2.7(3)$ & $\mathrm{C} 17-\mathrm{C} 18-\mathrm{C} 26-\mathrm{O} 4$ & $-1.2(3)$ \\
\hline $\mathrm{C} 13-\mathrm{C} 14-\mathrm{C} 15-\mathrm{C} 16$ & $-1.7(3)$ & $\mathrm{C} 19-\mathrm{C} 18-\mathrm{C} 26-\mathrm{C} 25$ & $-0.9(3)$ \\
\hline $\mathrm{C} 11-\mathrm{C} 14-\mathrm{C} 15-\mathrm{C} 16$ & $179.83(16)$ & $\mathrm{C} 17-\mathrm{C} 18-\mathrm{C} 26-\mathrm{C} 25$ & $179.60(17)$ \\
\hline $\mathrm{O} 3-\mathrm{C} 8-\mathrm{C} 16-\mathrm{C} 15$ & $179.29(18)$ & $\mathrm{N} 6-\mathrm{C} 25-\mathrm{C} 26-\mathrm{O} 4$ & $1.5(3)$ \\
\hline $\mathrm{C} 9-\mathrm{C} 8-\mathrm{C} 16-\mathrm{C} 15$ & $2.1(3)$ & $\mathrm{C} 21-\mathrm{C} 25-\mathrm{C} 26-\mathrm{O} 4$ & $-177.55(18)$ \\
\hline $\mathrm{O} 3-\mathrm{C} 8-\mathrm{C} 16-\mathrm{C} 17$ & $1.0(3)$ & $\mathrm{N} 6-\mathrm{C} 25-\mathrm{C} 26-\mathrm{C} 18$ & $-179.29(18)$ \\
\hline $\mathrm{C} 9-\mathrm{C} 8-\mathrm{C} 16-\mathrm{C} 17$ & $-176.17(18)$ & $\mathrm{C} 21-\mathrm{C} 25-\mathrm{C} 26-\mathrm{C} 18$ & $1.6(3)$ \\
\hline $\mathrm{C} 14-\mathrm{C} 15-\mathrm{C} 16-\mathrm{C} 8$ & $-0.3(3)$ & $\mathrm{C} 31-\mathrm{N} 7-\mathrm{C} 27-\mathrm{C} 28$ & $-1.7(3)$ \\
\hline $\mathrm{C} 14-\mathrm{C} 15-\mathrm{C} 16-\mathrm{C} 17$ & $177.9(2)$ & $\mathrm{C} 31-\mathrm{N} 7-\mathrm{C} 27-\mathrm{C} 17$ & $177.57(18)$ \\
\hline
\end{tabular}




$\begin{array}{llll}\mathrm{C} 8-\mathrm{C} 16-\mathrm{C} 17-\mathrm{C} 18 & -88.6(2) & \mathrm{C} 18-\mathrm{C} 17-\mathrm{C} 27-\mathrm{N} 7 & -35.5(2) \\ \mathrm{C} 15-\mathrm{C} 16-\mathrm{C} 17-\mathrm{C} 18 & 93.1(2) & \mathrm{C} 16-\mathrm{C} 17-\mathrm{C} 27-\mathrm{N} 7 & 92.9(2) \\ \mathrm{C} 8-\mathrm{C} 16-\mathrm{C} 17-\mathrm{C} 27 & 142.58(19) & \mathrm{C} 18-\mathrm{C} 17-\mathrm{C} 27-\mathrm{C} 28 & 143.83(19) \\ \mathrm{C} 15-\mathrm{C} 16-\mathrm{C} 17-\mathrm{C} 27 & -35.7(3) & \mathrm{C} 16-\mathrm{C} 17-\mathrm{C} 27-\mathrm{C} 28 & -87.8(2) \\ \mathrm{C} 27-\mathrm{C} 17-\mathrm{C} 18-\mathrm{C} 26 & -101.2(2) & \mathrm{N} 7-\mathrm{C} 27-\mathrm{C} 28-\mathrm{C} 29 & 2.8(3) \\ \mathrm{C} 16-\mathrm{C} 17-\mathrm{C} 18-\mathrm{C} 26 & 130.17(19) & \mathrm{C} 17-\mathrm{C} 27-\mathrm{C} 28-\mathrm{C} 29 & -176.4(2) \\ \mathrm{C} 27-\mathrm{C} 17-\mathrm{C} 18-\mathrm{C} 19 & 79.3(2) & \mathrm{C} 27-\mathrm{C} 28-\mathrm{C} 29-\mathrm{C} 30 & -1.3(4) \\ \mathrm{C} 16-\mathrm{C} 17-\mathrm{C} 18-\mathrm{C} 19 & -49.3(2) & \mathrm{C} 28-\mathrm{C} 29-\mathrm{C} 30-\mathrm{C} 31 & -1.2(4) \\ \mathrm{C} 26-\mathrm{C} 18-\mathrm{C} 19-\mathrm{C} 20 & -0.6(3) & \mathrm{C} 27-\mathrm{N} 7-\mathrm{C} 31-\mathrm{C} 30 & -1.0(3) \\ \mathrm{C} 17-\mathrm{C} 18-\mathrm{C} 19-\mathrm{C} 20 & 178.93(18) & \mathrm{C} 29-\mathrm{C} 30-\mathrm{C} 31-\mathrm{N} 7 & 2.5(4)\end{array}$

Hydrogen-bond geometry $\left(A,{ }^{\circ}\right)$

$C g 1, C g 2$ and $C g 3$ are the centroids of the $\mathrm{C} 18-\mathrm{C} 21 / \mathrm{C} 25 / \mathrm{C} 26, \mathrm{C} 8 / \mathrm{C} 9 / \mathrm{C} 13-\mathrm{C} 16$ and $\mathrm{N} 7 / \mathrm{C} 27-\mathrm{C} 31$ rings, respectively.

\begin{tabular}{lllll}
\hline$D-\mathrm{H} \cdots A$ & $D-\mathrm{H}$ & $\mathrm{H} \cdots A$ & $D \cdots A$ & $D-\mathrm{H} \cdots A$ \\
\hline $\mathrm{O} 3-\mathrm{H} 3 \cdots \mathrm{N} 5$ & $0.82(2)$ & $2.23(3)$ & $2.727(2)$ & $119(2)$ \\
$\mathrm{O} 3-\mathrm{H} 3 \cdots \mathrm{O} 4{ }^{\mathrm{i}}$ & $0.83(3)$ & $2.26(3)$ & $3.044(2)$ & $159(2)$ \\
$\mathrm{O} 4-\mathrm{H} 4 \cdots \mathrm{N} 5^{\mathrm{i}}$ & $0.84(4)$ & $2.16(3)$ & $2.878(3)$ & $144(2)$ \\
$\mathrm{O} 4-\mathrm{H} 4 \cdots \mathrm{N} 6$ & $0.84(4)$ & $2.20(4)$ & $2.674(3)$ & $115(3)$ \\
$\mathrm{C} 29-\mathrm{H} 29 \cdots \mathrm{O} 33^{\mathrm{ii}}$ & 0.95 & 2.53 & $3.377(3)$ & 148 \\
$\mathrm{C} 10-\mathrm{H} 10 \cdots C g 1^{\mathrm{iii}}$ & 0.95 & 2.75 & $3.659(3)$ & 161 \\
$\mathrm{C} 22-\mathrm{H} 22 \cdots C g 2^{\mathrm{i}}$ & 0.95 & 2.92 & $3.667(3)$ & 136 \\
$\mathrm{C} 24-\mathrm{H} 24 \cdots C g 3^{\mathrm{v}}$ & 0.95 & 2.65 & $3.528(3)$ & 153 \\
$\mathrm{C} 30-\mathrm{H} 30 \cdots C g 1^{\mathrm{ii}}$ & 0.95 & 2.73 & $3.562(3)$ & 147
\end{tabular}

Symmetry codes: (i) $-x+1,-y+2,-z+2$; (ii) $x-1, y, z$; (iii) $x+1, y+1, z$; (iv) $x, y-1, z$; (v) $-x,-y+1,-z+2$. 